\title{
The Effects of New Selective PPAR $\alpha$ Agonist CP775146 on Systematic Lipid Metabolism in Obese Mice and Its Potential Mechanism
}

\author{
Shengjie Tang, ${ }^{1}$ Fang Wu, ${ }^{1}$ Xihua Lin, ${ }^{2}$ Weiwei Gui, ${ }^{1}$ Fenping Zheng, ${ }^{1}$ and Hong Li $^{1}{ }^{1}$ \\ ${ }^{1}$ Department of Endocrinology, The Affiliated Sir Run Run Shaw Hospital, School of Medicine, Zhejiang University, Hangzhou, China \\ 310016 \\ ${ }^{2}$ Biomedical Research Center and Key Laboratory of Biotherapy of Zhejiang Province, The Affiliated Sir Run Run Shaw Hospital, \\ Zhejiang University, Hangzhou, Zhejiang, China 310016
}

Correspondence should be addressed to Hong Li; srrshnfm@zju.edu.cn

Received 20 January 2020; Revised 11 March 2020; Accepted 3 April 2020; Published 5 May 2020

Academic Editor: Bernd Stratmann

Copyright (C) 2020 Shengjie Tang et al. This is an open access article distributed under the Creative Commons Attribution License, which permits unrestricted use, distribution, and reproduction in any medium, provided the original work is properly cited.

Purpose. Peroxisome proliferator-activated receptor $\alpha(\mathrm{PPAR} \alpha)$ plays a crucial role in the control of lipid homeostasis. Here, we investigated the effects of CP775146, a new selective PPAR $\alpha$ agonist, on lipid metabolism in diet-induced obese mice and its possible mechanism. Methods. C57BL/6 mice were fed a high-fat diet (HFD) for 12 weeks to induce obesity and then received CP775146 via intraperitoneal injection for 3 days. The content/morphology of the liver, serum lipid, and liver function was measured. The expression of genes related to lipolysis and synthesis in liver was detected by quantitative real-time PCR (qRTPCR). Results. The safe dose of CP775146 was $<0.3 \mathrm{mg} / \mathrm{kg}$. CP775146 reduced the serum levels of liver enzymes, such as alanine aminotransferase (ALT) and glutamic-oxaloacetic transaminase (AST) and lipid metabolism-related biomarkers, including triglycerides (TGs) and low-density lipoprotein cholesterol (LDL-c), non-high-density lipoprotein cholesterol (non-HDL-c), and hepatic TG content, at a dosage of $0.1 \mathrm{mg} / \mathrm{kg}$. HFD-induced pathological liver changes improved after CP775146 treatment. The expression of genes involved in liver fatty acid oxidation (acyl-coenzyme A dehydrogenase, long chain (Acadl), acyl-CoA oxidase 1 (Acox-1), carnitine palmitoyltransferase-1 (CPT-1), and enoyl-CoA, hydratase/3-hydroxyacyl CoA dehydrogenase (Ehhadh)) was upregulated in CP775146-treated mice. Furthermore, CP775146 induced the expression of thermogenesis genes (cell death-inducing DFFA-like effector a (Cidea), uncoupling protein 1 (Ucp1)) and lipolysis genes (hormone-sensitive lipase $(H s l)$, adipose tissue triglyceride lipase $(A t g l)$ ) in epididymal white adipose tissue (eWAT), activating browning and thermogenesis. Conclusion. CP775146 efficiently alleviates obesity-induced liver damage, prevents lipid accumulation by activating the liver fatty acid $\beta$-oxidation pathway, and regulates the expression of genes that control brown fat-like pathway in eWAT.

\section{Introduction}

Dyslipidemic conditions are characterized by increased levels of blood free fatty acid (FFA), triglyceride (TG), total cholesterol (TC), and low-density lipoprotein cholesterol (LDL-c) and decreased levels of high-density lipoprotein cholesterol (HDL-c) [1]. Dyslipidemia has been linked to the increased prevalence of diabetes, cardiovascular diseases, and fatty liver diseases [2-4]. In addition to genetic factors associated with familial lipid disorders, dyslipidemia is closely linked to the dysregulation of nutrient homeostasis, which defines obesity. Abnormal adipose tissues secrete several adipokines and produce excessive FFAs, thereby enhancing dyslipidemia [5]. Hence, lipid control therapies are urgently required.

Peroxisome proliferator-activated receptors (PPARs) are members of the superfamily of nuclear hormone receptors that were first discovered in Xenopus [6]. Upon ligand binding, PPARs (PPAR $\alpha$, PPAR $\gamma$, and PPAR $\delta / \beta)$ form heterodimers with retinoid $\mathrm{X}$ receptors and then interact with PPAR response elements to regulate target gene expression [7]. The 
three PPAR subtypes are expressed differentially in various tissues, thereby allowing selective changes in the expression levels of genes related to lipid and glucose metabolism. Tissues actively engage in fatty acid metabolism, such as the liver, brown adipose tissue (BAT), and the heart [8], and express high levels of $\operatorname{PPAR} \alpha$, which play major roles in fatty acid uptake and activation of mitochondrial $\beta$-oxidation, glucose metabolism, and hepatic acute phase response [9]. $\operatorname{PPAR} \alpha$ regulates the expression of genes encoding the ratelimiting enzymes of peroxisomal $\beta$-oxidation, including Acoxl and Ehhadh. Fatty acid transport across the mitochondrial membrane is triggered by $\operatorname{PPAR} \alpha$-dependent regulation of carnitine palmitoyltransferase-1 (CPT-1) [10]. Thus, PPAR $\alpha$ is the primary target of hypolipidemic drugs, including fibrates (e.g., gemfibrozil, bezafibrate, and fenofibrate). However, these agents are weak and relatively poorly selective PPAR agonists and often trigger muscle or cardiac toxicities [11]. An efficient and selective $\operatorname{PPAR} \alpha$ agonist is urgently required.

CP775146 is a novel piperidine-based PPAR $\alpha$ agonist that binds more strongly and selectively to the PPAR $\alpha$ ligand than classical fibrates. CP775146 exhibited outstanding TGlowering activity in chow-fed mice and activated transcriptional networks triggering $\operatorname{PPAR} \alpha$-mediated induction of fatty acid oxidation and anti-inflammatory activities [12]. However, this drug is used to treat patients with hyperlipidemia. The effects of CP775146 on lipid metabolism in obese mice remain unknown.

This study was conducted to evaluate the effects of CP775146 on systematic lipid metabolism and its potential mechanism, in diet-induced obese mice and focused on $\operatorname{PPAR} \alpha$ target tissues.

\section{Materials and Methods}

2.1. Animals, Diets, and Treatments. Four-week-old male C57BL/6 mice were purchased from the Slack Experimental Animal Center of the Chinese Academy of Sciences (Shanghai, China). Mice were housed at $22^{\circ} \mathrm{C}$ under a $12 \mathrm{~h} / 12 \mathrm{~h}$ light/dark cycle and given free access to water and standard chow $(63.92 \%$ carbohydrates, $26.18 \%$ protein, and $9.9 \%$ fat) or a high-fat diet (HFD; 35\% carbohydrates, $20 \%$ protein, and $45 \%$ fat) for 12 weeks. The safe dose of CP775146 was determined. Chow-fed mice were randomized into the following groups: (1) chow-control $(n=4)$ and (2) chowCP775146 groups $(0.3,0.6,1.0,1.2$, or $1.5 \mathrm{mg} / \mathrm{kg}) ; n=4$ / group. According to the safe dose, HFD-fed mice were also divided into the following groups: (1) HFD-NC $(n=4),(2)$ HFD-0.1 (0.1 mg/kg, $\mathrm{n}=4)$, and (3) HFD-0.3 (0.3 mg/kg, $n=4)$. Saline or CP775146 was given via intraperitoneal injection for 3 days. After fasting for $12 \mathrm{~h}$ since the last drug treatment, all mice were killed via $\mathrm{CO}_{2}$ asphyxiation. Trunk blood was collected for biochemical analyses. The selective PPAR $\alpha$ agonist CP775146 (purity $\geq 98 \%$ ) was obtained from Sigma Chemical Co. (St. Louis, MO, USA).

All animal experimental procedures were approved by the Animal Welfare Ethics Committee of Zhejiang University.
2.2. Serum Biochemical Measurements. Blood was held at room temperature for $30 \mathrm{~min}$ and centrifuged to obtain serum, which was stored at $-80^{\circ} \mathrm{C}$ prior to analysis. The levels of TC, TG, LDL-c, HDL-c, and liver enzyme were measured automatically (Hitachi 7020, Japan). Non-HDL-c values were calculated as follows: non $-\mathrm{HDL}-\mathrm{c}=\mathrm{TC}-(\mathrm{HDL}-\mathrm{c})$.

2.3. Histological Study of Liver Tissue and Epididymal White Adipose Tissue (eWAT). Liver tissue and eWAT were weighed and fixed. The samples were then embedded in paraffin blocks, sectioned at a thickness of $5 \mu \mathrm{m}$, stained with hematoxylin and eosin (H\&E), and observed under a light microscope.

2.4. Hepatic TG and TC Measurements. Hepatic TG and TC levels were measured using $100 \mathrm{mg}$ of frozen liver samples. The tissues were homogenized in $1 \mathrm{~mL}$ of phosphatebuffered saline $(1 \mathrm{x})$ and centrifuged at 2,500 rpm. The supernatant $(20 \mu \mathrm{L})$ was assayed using commercial kits (Nanjing Jiancheng, China) according to the manufacturer's protocols.

2.5. Quantitative Real-Time PCR. Total RNA was extracted from liver tissues by using RNAiso Plus (Takara Bio Inc., Shiga, Japan) according to the manufacturer's instructions and stored at $-80^{\circ} \mathrm{C}$ prior to analysis. First-strand cDNA synthesis was performed according to the manufacturer's instructions, followed by PCR. Data were quantitated using the relative mRNA expression ratio $\left(2^{-\Delta \Delta c t}\right.$ method). Primer sequences are listed in Table 1.

2.6. Western Blot Analysis. Total protein was isolated from the liver in a lysis buffer for $30 \mathrm{~min}$ at $4^{\circ} \mathrm{C}$. Protein concentration was measured using BCA Protein Assay Reagent (P0011, Beyotime Biotechnology, China). The proteins were transferred to a polyvinylidene difluoride membrane, blocked with 5\% nonfat dry milk in PBS with $0.02 \%(v / v)$ Tween20 , and incubated with primary antibodies at $4^{\circ} \mathrm{C}$ overnight. The membrane was washed and incubated for $1 \mathrm{~h}$ at room temperature with a peroxidase-labeled secondary antibody. After washing, protein bands were visualized by electrochemiluminescence (FD8030, FDBio Science, China). Anti$\operatorname{Pgc1} \beta$ (A17089), Cpt1 $\alpha$ (A5307), and Cpt1 $\beta$ (A6796) were obtained from ABclonal (Wuhan, China). Mouse anti- $\beta$ actin antibody (A5441) was purchased from Sigma-Aldrich (St. Louis, MO, USA).

2.7. Statistical Analysis. Data are presented as means \pm SD. Statistical analysis was conducted with SPSS version 20.0 software by using one-way ANOVA for multiple group comparisons or Student's $t$-test for two-group comparisons. $P$ values $<0.05$ indicated statistical significance.

\section{Results}

3.1. CP775146 Attenuates Dyslipidemia in HFD-Induced Obese Mice. Chow-fed mice were treated with different doses of CP775146. CP775146 increased the liver weight but did not affect the on mean body weight (Figures 1(a) and 1(b)). At $>0.3 \mathrm{mg} / \mathrm{kg}, \mathrm{CP} 775146$ significantly increased the serum 
TABle 1: Primer sequences of real-time polymerase chain reaction.

\begin{tabular}{|c|c|c|}
\hline Gene & Forward & Reverse \\
\hline Acadl & AGGGTTTAGTTTTGAGTTGACGG & CCCCGCTTTTGTCATATTCCG \\
\hline Acadm & TCTTTTCCTCGGAGCATGACA & GACСТСТСТАСТСАСТTСТССАG \\
\hline Acoxl & CGATCCAGACTTCCAACATGAG & CCATGGTGGCACTCTTCTTAACA \\
\hline Cpt1a & CTCCGCCTGAGCCATGAAG & CACCAGTGATGATGCCATTCT \\
\hline Cpt1b & GCACACCAGGCAGTAGCTTT & CAGGAGTTGATTCCAGACAGGT \\
\hline$F g f 21$ & AGATCAGGGAGGATGGAACA & TCAAAGTGAGGCGATCCATA \\
\hline Ehhadh & ATGGCTGAGTATC TGAGGCTG & ACCGTATGGTCCAAACTAGCTT \\
\hline $\mathrm{CrebH}$ & CCTGTTTGATCGGCAGGAC & CGGGGGACGATAATGGAGA \\
\hline PPAR $\alpha$ & GCAGTGCCCTGAACATCGA & CGCCGAAAGAAGCCCTTAC \\
\hline Ucp1 & GGCATTCAGAGGCAAATCAGCT & CAATGAACACTGCCACACCTC \\
\hline Hsl & GAGCGCTGGAGGAGTGTTTT & TGATGCAGAGATTCCCACCTG \\
\hline Atgl & GGATGGCGGCATTTCA & CAAAGGGTTGGGTTGG \\
\hline Cidea & GCAGCCTGCAGGAACTTATCAGC & GATCATGAAATGCGTGTTGTCC \\
\hline$\beta$-Actin & GAGACCTTCAACACCCCAGC & ATGTCACGCACGATTTCCC \\
\hline
\end{tabular}

alanine aminotransferase (ALT) level (a measure of liver function, Figure 1(c)) but not that of aspartate aminotransferase (AST, Figure 1(d)). H\&E staining showed that the hepatocytes of the groups $(>0.3 \mathrm{mg} / \mathrm{kg})$ contained more lipid droplets and exhibited more ballooning-induced degeneration than those of the control and $0.3 \mathrm{mg} / \mathrm{kg}$ group, respectively (Figure 1(e)). Thus, CP775146 at $>0.3 \mathrm{mg} / \mathrm{kg}$ was hepatotoxic.

The mean body weight (Figure 2(a)) of the HFD mice was higher than that of chow-fed mice, but CP775146 had no effect on the body weight. CP775146-treated mice exhibited significantly higher liver/body weight ratio (Figure 2(b)) than the HFD-NC group. In terms of serum biochemical parameters (Figures 2(c)-2(f)), HFD-NC mice had higher TG, TC, LDL-c, HDL-c, and non-HDL-c levels than chow-fed mice did (Figures 2(c)-2(g)). The plasma levels of TG, LDL-c, and non-HDL-c in the HFD-CP775146 group (Figures 2(c), $2(\mathrm{e})$, and 2 (g), respectively) were significantly lower than those tin the HFD-NC group in a CP775146 dose-dependent manner. CP775146-treated mice exhibited a trend toward a decrease in the serum TC level (Figure 2(d)) but not HDL-c level (Figure 2(f)).

3.2. CP775146 Alleviates Hepatic Damage Induced by HFD. $\mathrm{H} \& \mathrm{E}$ staining showed that hepatocytes in the HFD-NC group (Figure 3(b)) were larger than those in the chowfed group (Figure 3(a)), contained increased amounts of lipid droplets, and exhibited additional ballooning-induced degeneration (Figure 3(b)). CP775146 alleviated HFDinduced pathological liver changes (Figures 3(c) and 3(d)). CP775146 reduced the hepatic TG levels at a lower dose $(0.1 \mathrm{mg} / \mathrm{kg})$ than those in HFD-NC mice (Figure 3(e)). CP775146 tended to decrease the hepatic TC levels (Figure 3(f)) but not significantly. HFD-NC mice exhibited increased serum ALT and AST levels relative to chow-fed mice (Figures 3(g) and 3(h)). CP775146 at $0.1 \mathrm{mg} / \mathrm{kg}$ significantly inhibited HFD-induced increases in ALT and AST levels (Figures 3(g)and 3(h)).
3.3. CP775146 Affects the Expression of Genes Involved in Liver Lipid Metabolism. CP775146 did not affect the liver level of mRNA encoding PPAR $\alpha$ compared with that in the HFD-NC group. However, CP775146 significantly increased the expression levels of PPAR $\alpha$ target genes responsible for fatty acid oxidation, including Acadl, Acoxl, and Ehhadh(Figure 3(i)). CP775146 significantly increased the expression levels of $F g f 21$ and Cpt1 $\beta$ (Figure 3(j)). CP775146 also upregulated the protein levels of $\mathrm{Cpt} 1 \alpha$ and $C p t 1 \beta$ (Figures 3, K1 and K2). Thus, CP775146 activated fatty acid oxidation and PPAR $\alpha$ signaling in the livers of HFD-fed mice.

3.4. CP775146 Activates PPAR $\alpha$-Mediated Gene Expression in eWAT of HFD-Fed Mice. The H\&E staining of the eWAT showed that $0.1 \mathrm{mg} / \mathrm{kg}$ CP775146 decreased the adipocyte sizes compared with HFD-NC mice (Figures 4(a)-4(c)), and no change was observed in the HFD-0.3 group (Figure 4(d)). In the eWAT, CP775146 significantly increased the expression levels of adipose TG lipase (ATGL) and hormone-sensitive lipase ( $H s l$; Figure 4(e)), thereby inducing lipolysis. CP775146 also upregulated the expression of genes involved in thermogenesis, including Ucp1 and Cidea but did not change that of PPAR $\alpha$ compared with that in HFD-NC mice (Figure 4(e)).

\section{Discussion}

We explored the effects of CP775146, a new selective PPAR $\alpha$ agonist, on lipid metabolism in diet-induced obese mice. CP775146 significantly reduced the plasma TG and LDL-c levels and hepatic TG content at the dosage of $0.1 \mathrm{mg} / \mathrm{kg}$. CP775146 reduced the plasma ALT levels and pathological liver changes, thereby alleviating hepatic damage induced by HFD. CP775146 activated the liver PPAR $\alpha$-associated pathway of fatty acid oxidation and upregulated the expression genes involved in thermogenesis and lipolysis in eWAT. Hence, CP775146 improved systematic lipid metabolism in HFD-induced obese mice. 


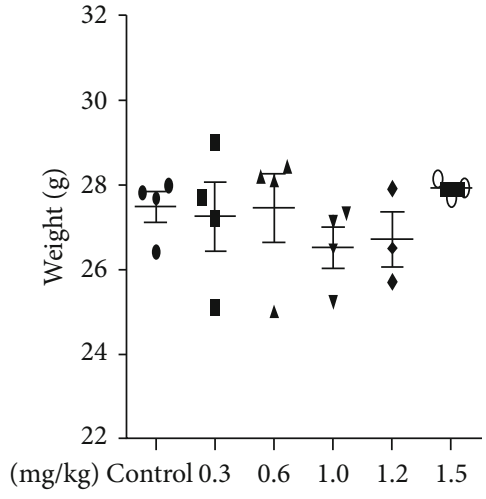

(a)

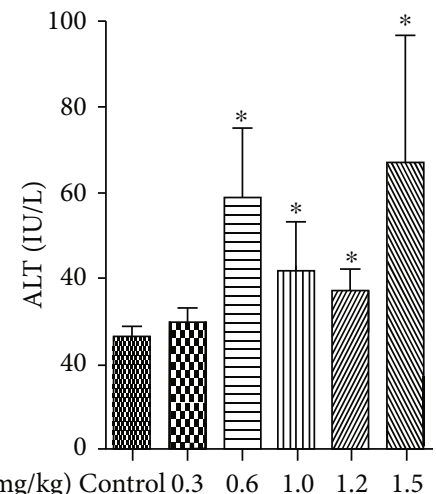

(c)

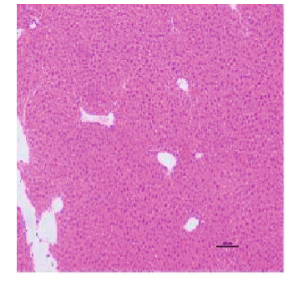

Control

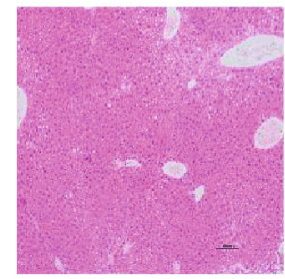

1.0

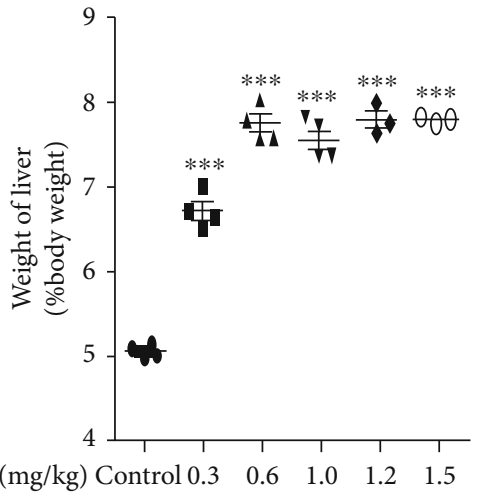

(b)

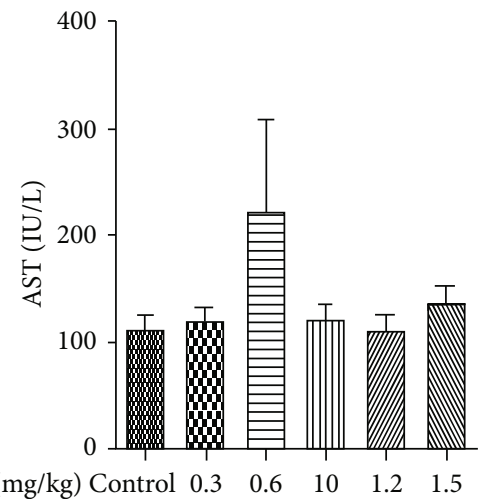

(d)

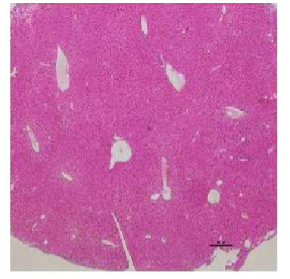

0.6

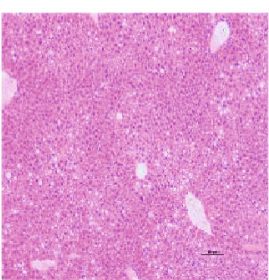

1.2

(e)

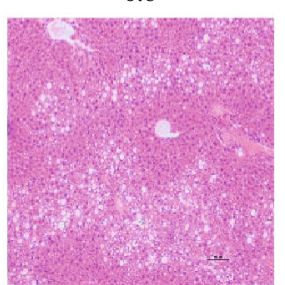

1.5

FIgure 1: The safe dose of CP775146. (a) Body weight, (b) liver/weight ratio, (c) ALT level, and (d) AST level. (e) H\&E staining of the liver sections (scale bar $=100 \mu \mathrm{m}$ ). Means \pm SDs $\left(n=4\right.$ /group) are shown. ${ }^{*} P<0.05,{ }^{* *} P<0.01$, and ${ }^{* * *} P<0.001$ vs. the chow-fed control group.

PPAR $\alpha$ is mainly expressed in the liver and plays a crucial role in hepatic physiology by regulating the balance between systematic fatty acid and TG metabolism [13]. Compared with classical PPAR $\alpha$ agonists, CP775146 is a selective PPAR $\alpha$ modulator that strongly and selectively stimulates PPAR $\alpha$ related pathways [12]. CP775146 significantly upregulated the expression levels of the Acoxl, Acadl, and Ehhadh genes involved in fatty acid oxidation and decreased plasma and hepatic TG levels. In the liver, adipose tissues, and normal lipogenic tissues, fatty acid pathways facilitate the storage of excess energy as TG, which is later used to supply energy. Fatty acid oxidation occurs in the mitochondria, and CPT-1 is the key enzyme that regulates the entry of fatty acids into the mitochondria [14]. PPAR $\alpha$ upregulates $C P T-1$ [15]. Mammals have three CPT1 isoforms (CPT1A, CPT1B, and $C P T 1 C)$. CPT1A is enriched in the liver and plays a key role in fatty acid oxidation. $C P T 1 B$ is mainly found in muscles, including cardiomyocytes, which are the major consumers 


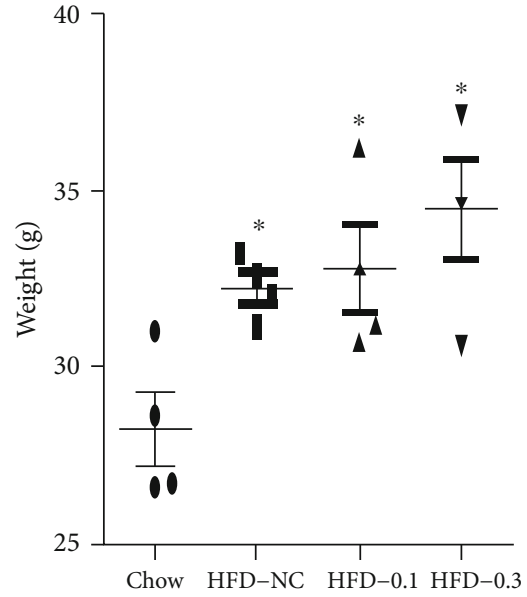

(a)

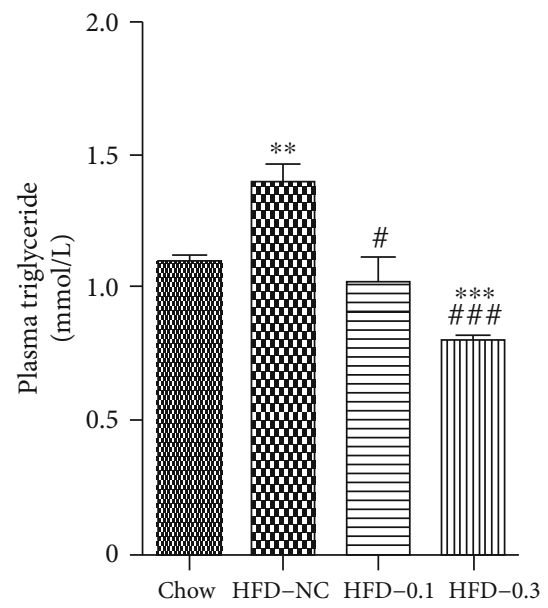

(c)

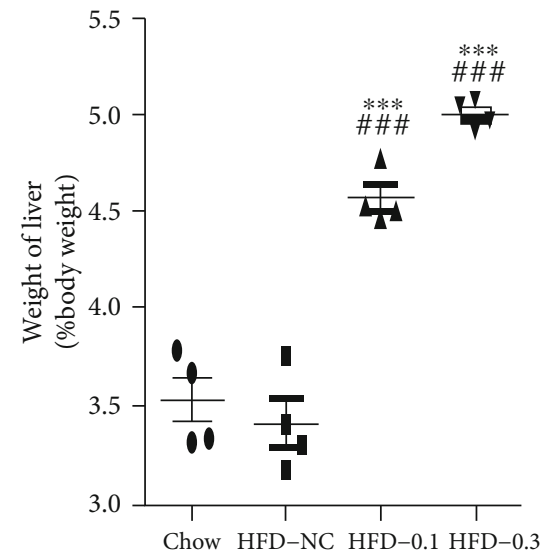

(b)

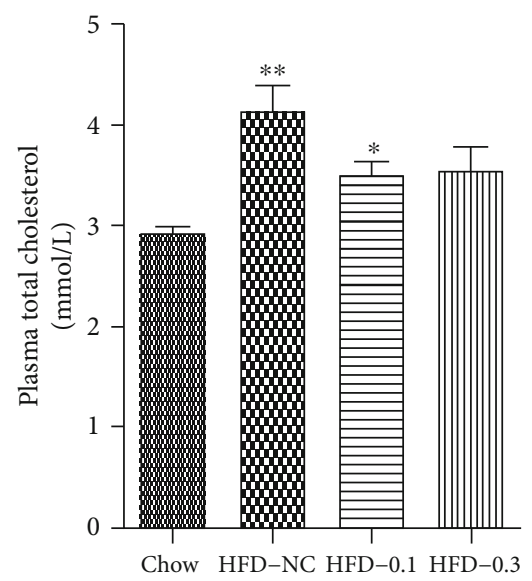

(d)

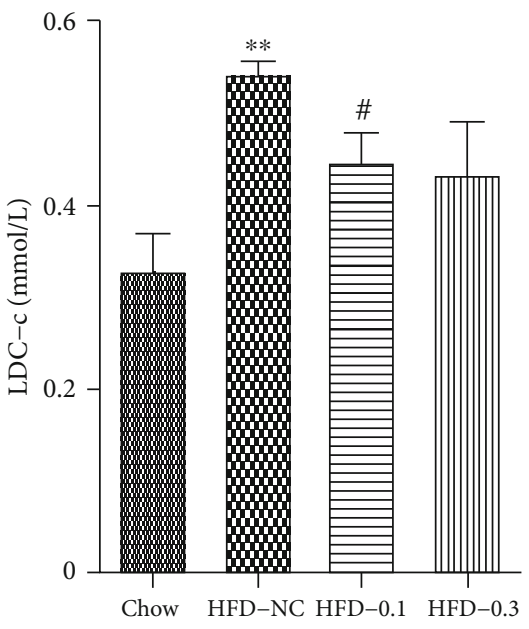

(e)

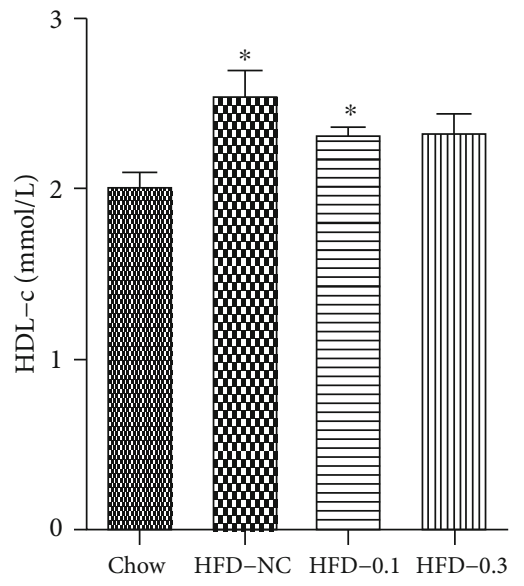

(f)

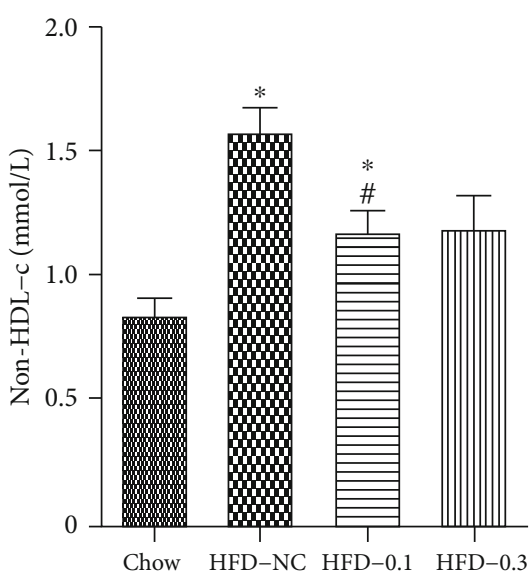

(g)

FIGURE 2: CP775146 attenuates dyslipidemia in HFD mice. (a) Body weight, (b) liver/weight ratio, (c) serum TG level, (d) serum TC level, (e) serum LDL-c level, (f) serum HDL-c level, and (g) serum non-HDL-c level. Means \pm SDs ( $n=4$ /group) are shown. ${ }^{*} P<0.05$, ${ }^{* *} P<0.01$, and ${ }^{* * *} P<0.001$ vs. chow-fed mice; ${ }^{\#} P<0.05$, ${ }^{\# \#} P<0.01$, and ${ }^{\# \# \# ~} P<0.001$ vs. the HFD-NC group. According to the safe dose, HFD-fed mice were divided into these groups: (1) HFD-NC group, $n=4$; (2) HFD-0.1 group (0.1 mg/kg), $n=4$; and (3) HFD-0.3 group (0.3 mg/kg), $n=4$. Saline or CP775146 was given via intraperitoneal injection for 3 days. 

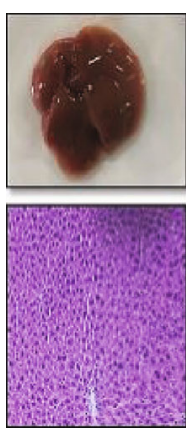

(a)

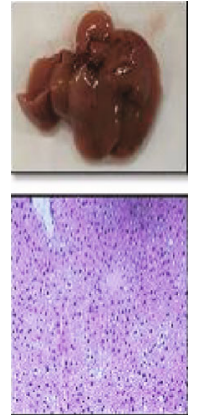

(b)

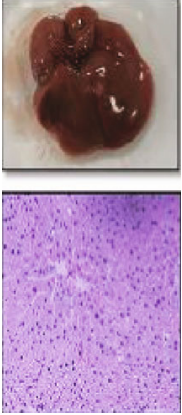

(c)

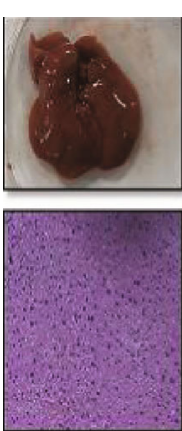

(d)

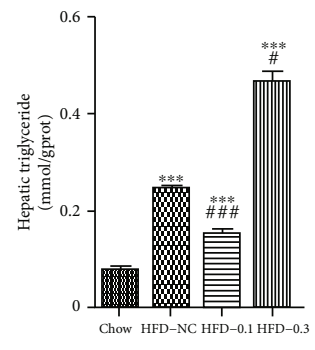

(e)

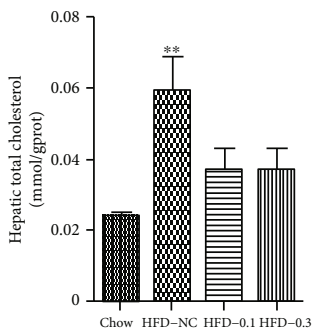

(f)

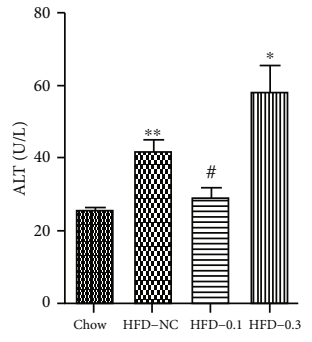

(g)

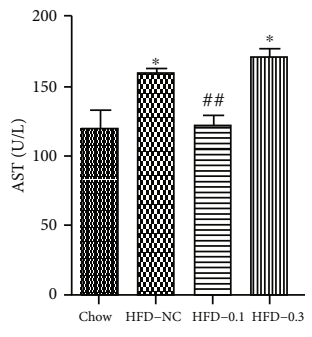

(h)

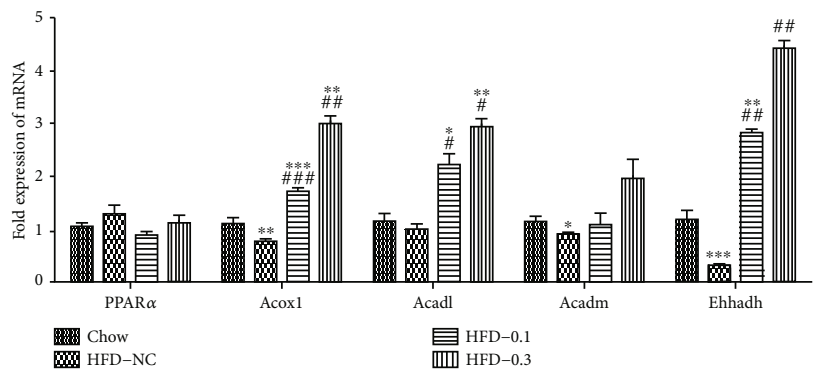

(i)

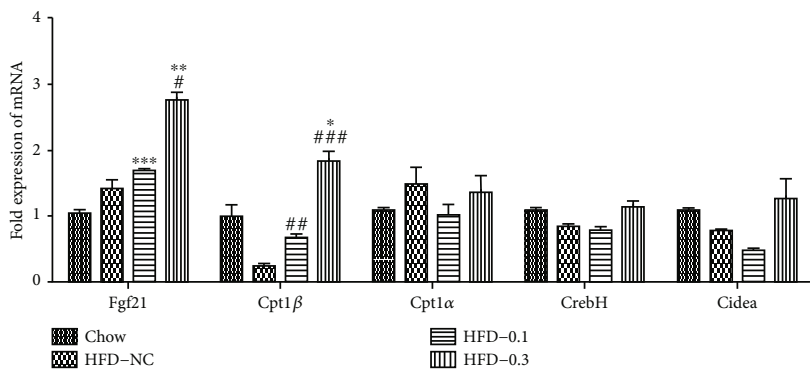

(j)

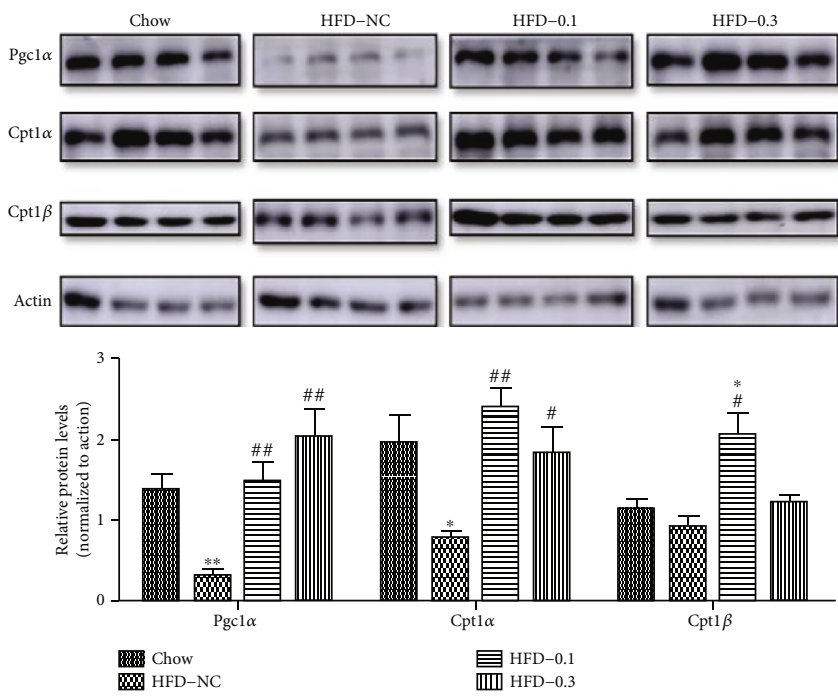

(k)

Figure 3: CP775146 alleviates hepatic damage induced by an HFD. H\&E staining of liver sections (original magnification, 200x); $n=4$ /group. (a) Chow-fed group, (b) HFD-NC group, (c) HFD-CP775146 $0.1 \mathrm{mg} / \mathrm{kg}$ group, and (d) HFD-CP775146 $0.3 \mathrm{mg} / \mathrm{kg}$ group. (e) TG and (f) TC liver levels, (g) ALT levels, and (h) AST levels. $(\mathrm{i}, \mathrm{j})$ The mRNA expression levels of various genes. Means \pm SDs are shown ( $n=4 / \mathrm{group})$. (K1, K2) The protein expression levels of various genes. ${ }^{*} P<0.05$, ${ }^{* *} P<0.01$, and ${ }^{* * *} P<0.001$ vs. the chow-fed group; ${ }^{\#} P<0.05$, ${ }^{\# \#} P<0.01$, and ${ }^{\# \# \#} P<0.001$ vs. HFD-NC group. 


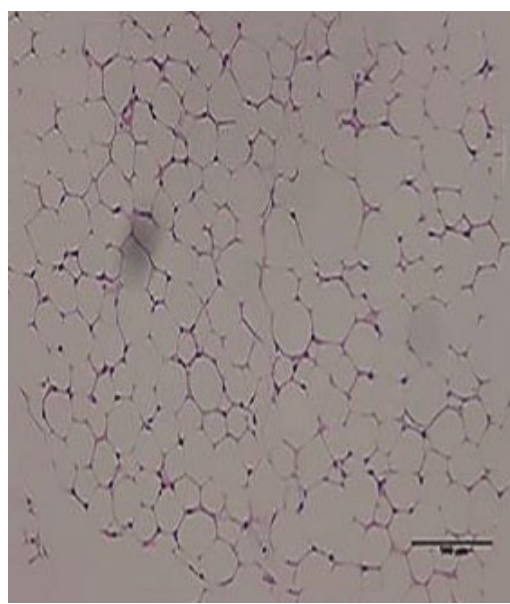

(a)

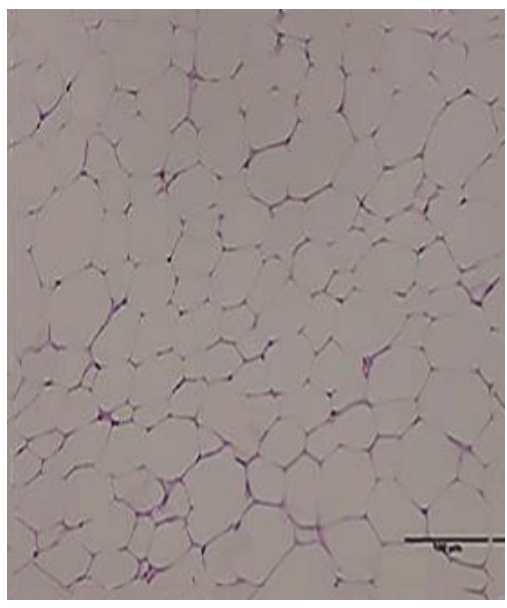

(c)

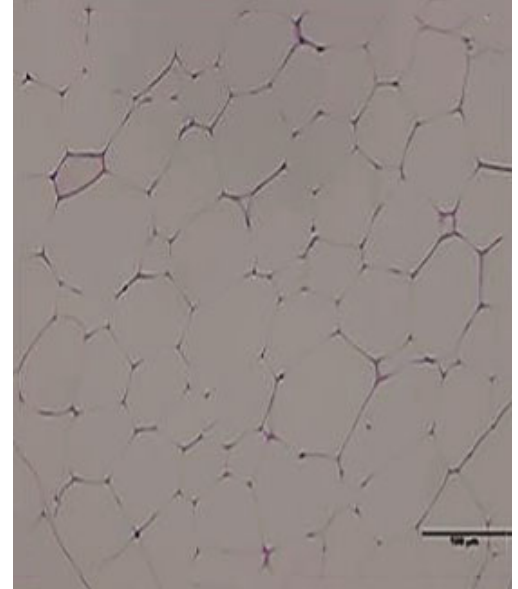

(b)

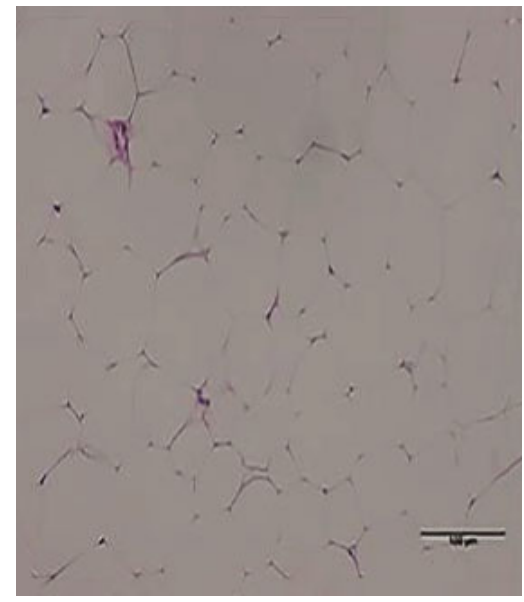

(d)

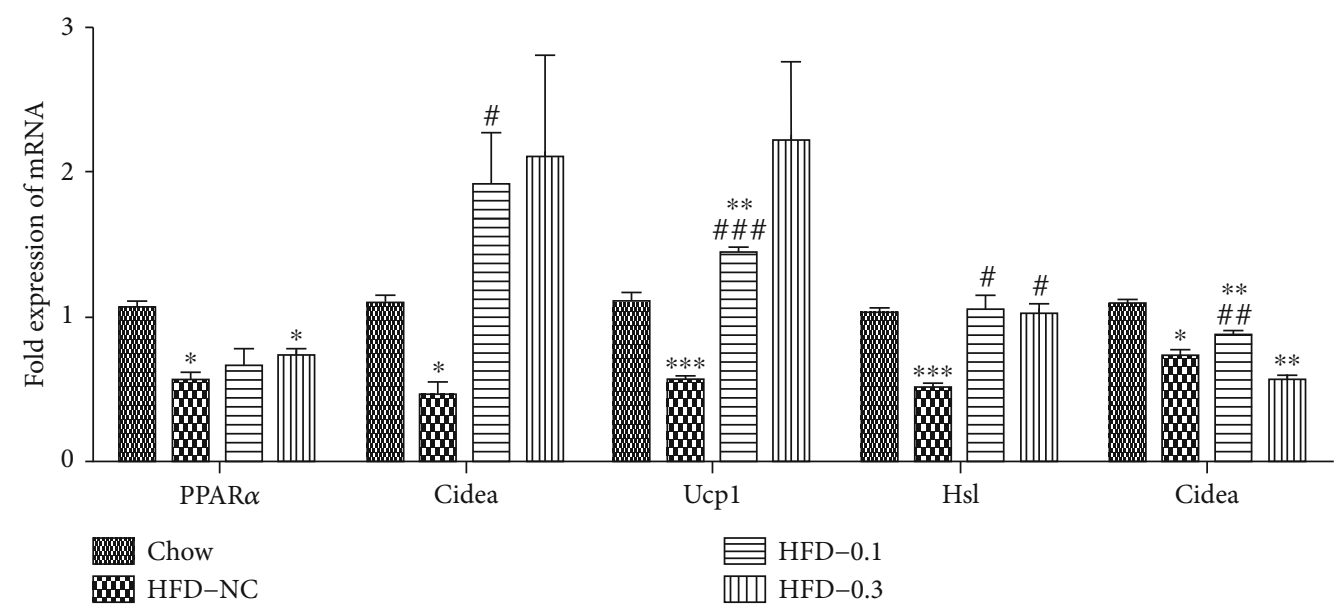

(e)

FIgURE 4: CP775146 activates PPAR $\alpha$-mediated gene expression in the eWAT of mice fed an HFD. eWAT H\&E staining data, scale bar $=100$ $\mu \mathrm{m} ; n=4$ /group. (a) Chow-fed group, (b) HFD-NC group, (c) HFD-CP775146 0.1 mg/kg group, and (d) HFD-CP775146 $0.3 \mathrm{mg} / \mathrm{kg}$ group. (e) The expression levels of genes involved in thermogenesis and lipolysis. Means \pm SDs are shown ( $n=4$ /group). ${ }^{*} P<0.05$, ${ }^{* *} P<0.01$, and ${ }^{* * *} P<0.001$ vs. the chow-fed group; ${ }^{\#} P<0.05,{ }^{\# \#} P<0.01$, and ${ }^{\# \# \#} P<0.001$ vs. HFD-NC group.

of fatty acids. CPT1C is present in the brain and testis [16]. A previous study suggested that the three isoforms are confined to specific tissues [17]. CPT1A, as the liver isoform, catalyzes the rate-limiting step of converting acyl-coenzyme into acyl-carnitines, which can cross the membranes to enter the mitochondria in the fatty acid oxidation pathway [18]. 
CPT1A protects obese mice against hepatic steatosis and insulin resistance [19]. In the present study, the level of mRNA encoding CPT1B was downregulated in the liver of HFD-fed mice. CP775146 reversed this effect and upregulated the expression levels of genes that are involved in fatty acid oxidation in HFD-fed mice. The protein levels of Cpt $1 \alpha$ and $\operatorname{Cpt} 1 \beta$ were also upregulated. $C p t 1 \beta$ may play a vital role in CP775146-mediated activation of fatty acid oxidation in the liver.

Adipocytes provide metabolic energy by balancing lipolysis and TG synthesis [20]. Lipolysis in the WAT is stimulated by $\beta 3$ adrenergic receptor signaling, which activates lipolytic enzymes, including HSL, ATGL, and perilipin [21]. HSL plays a major role in the lipolysis of cellular fat stores [22]. The activation of thermogenic genes, including Ucp1, Cidea, and Cpt1b, converts white adipocytes into BATlike beige adipocytes [23]. This activation process reduces WAT weight and lipid droplet size. In the present study, CP775146 activated the expression levels of eWAT genes that are involved in lipolysis ( $\mathrm{Hsl}$ and $\mathrm{Atgl}$ ) and thermogenesis (Ucp1 and Cidea). The mechanism by which CP775146 decreases adipocyte sizes remains unclear.

CP775146 significantly increased the liver weight in chow- and HFD-fed mice. A previous study showed that the liver/body weight ratio increases significantly after gemfibrozil treatment. $\operatorname{PPAR} \alpha$ activation plays a crucial role in hepatomegaly (pathological liver enlargement) induced by peroxisome proliferation $[24,25]$. In the present study, the increase in the serum liver enzyme levels can be partly attributed to mitochondrial overload, which in turn reflected the overactivation of fatty acid oxidation. CP775146 efficacy was compromised by dose-related adverse effects, which have gained increasing research attention. Further studies are required to explore these adverse reactions and the mechanism underlying their prevention.

\section{Conclusion}

CP775146, a new selective PPAR $\alpha$ agonist, significantly reduces the levels of serum TG and liver enzymes, improves hepatic steatosis induced by HFD, and decreases adipocyte droplet sizes. The effects of CP775146 are partially mediated through its regulation of $\operatorname{PPAR} \alpha$ target genes that are involved in fatty acid oxidation in the liver and lipolysis in eWAT. Further study on the mechanism of CP775146 on lipid metabolism may provide new sights into the clinical treatment of hyperlipidemia.
Abbreviation

Acox1: Acyl-CoA oxidase 1

Acadl: Acyl-coenzyme A dehydrogenase, long-chain

CPT-1: $\quad$ Carnitine palmitoyltransferase-1

Ehhadh: Enoyl-CoA, hydratase/3-hydroxyacyl CoA

Fgf21: $\quad$ Fibroblast growth factor 21

eWAT: $\quad$ Epididymal white adipose tissue

Cidea: Cell death-inducing DFFA-like effector a

Ucp1: Uncoupling protein 1

Hsl: $\quad$ Hormone-sensitive lipase

Atgl: $\quad$ Adipose tissue triglyceride lipase

CrebH: $\quad$ cAMP-responsive element-binding protein $\mathrm{H}$

FFA: $\quad$ Free fatty acid

RXR: $\quad$ Retinoid X receptor

PPRES: $\quad$ PPAR response element

BAT: $\quad$ Brown adipose tissue

H\&E: $\quad$ Hematoxylin and eosin

PBS: $\quad$ Phosphate-buffered saline

qRT-PCR: Quantitative real-time PCR.

\section{Data Availability}

The datasets used and/or analyzed during the present study are available from the corresponding author on reasonable request.

\section{Ethical Approval}

All applicable international, national, and/or institutional guidelines for the care and use of animals were followed.

\section{Conflicts of Interest}

The authors declare that no conflict of interest exists.

\section{Authors' Contributions}

Shengjie Tang and Fang Wu contributed equally to this work.

\section{Acknowledgments}

This study was supported by the National Natural Science Foundation of China (grant number 81873653), the National Natural Science Foundation of China for Youth (grant number 30800533) and the Zhejiang Provincial Medical Science and Technology Program (grant numbers 2017KY403 and 2015RCA013).

\section{References}

[1] X. Wang, L. Shi, S. Joyce, Y. Wang, and Y. Feng, "MDG-1, a potential regulator of $\operatorname{PPAR} \alpha$ and $\operatorname{PPAR} \gamma$, ameliorates dyslipidemia in mice," International Journal of Molecular Sciences, vol. 18, no. 9, article 1930, 2017.

[2] M. Castro Cabezas, B. Burggraaf, and B. Klop, "Dyslipidemias in clinical practice," Clinica Chimica Acta, vol. 487, pp. 117125, 2018.

[3] F. Landi, A. M. Martone, S. Salini et al., "Effects of a new combination of medical food on endothelial function and lipid profile in dyslipidemic subjects: a pilot randomized 
trial," BioMed Research International, vol. 2019, Article ID 1970878, 7 pages, 2019.

[4] E. Y. Kwon, S. Y. Kim, and M. S. Choi, "Luteolin-enriched artichoke leaf extract alleviates the metabolic syndrome in mice with high-fat diet-induced obesity," Nutrients, vol. 10, no. 8, p. 979, 2018.

[5] U. J. Jung and M. S. Choi, "Obesity and its metabolic complications: the role of adipokines and the relationship between obesity, inflammation, insulin resistance, dyslipidemia and nonalcoholic fatty liver disease," International Journal of Molecular Sciences, vol. 15, no. 4, pp. 6184-6223, 2014.

[6] I. Issemann and S. Green, "Activation of a member of the steroid hormone receptor superfamily by peroxisome proliferators," Nature, vol. 347, no. 6294, pp. 645-650, 1990.

[7] K. Takei, S. Han, Y. Murayama et al., "Selective peroxisome proliferator-activated receptor- $\alpha$ modulator K-877 efficiently activates the peroxisome proliferator-activated receptor- $\alpha$ pathway and improves lipid metabolism in mice," Journal of Diabetes Investigation, vol. 8, no. 4, pp. 446-452, 2017.

[8] J. Y. An, H. F. Jheng, H. Nagai et al., "A phytol-enriched diet activates PPAR- $\alpha$ in the liver and brown adipose tissue to ameliorate obesity-induced metabolic abnormalities," Molecular Nutrition \& Food Research, vol. 62, no. 6, 2018.

[9] M. Rakhshandehroo, L. M. Sanderson, M. Matilainen et al., "Comprehensive Analysis of PPAR $\alpha$-Dependent Regulation of Hepatic Lipid Metabolism by Expression Profiling," PPAR Research, vol. 2007, Article ID 26839, 13 pages, 2007.

[10] M. Rakhshandehroo, G. Hooiveld, M. Muller, and S. Kersten, "Comparative analysis of gene regulation by the transcription factor PPARalpha between mouse and human," PLoS One, vol. 4, no. 8, article e6796, 2009.

[11] A. Rubenstrunk, R. Hanf, D. W. Hum, J. C. Fruchart, and B. Staels, "Safety issues and prospects for future generations of PPAR modulators," Biochimica et Biophysica Acta (BBA) Molecular and Cell Biology of Lipids, vol. 1771, no. 8, pp. 1065-1081, 2007.

[12] C. D. Kane, K. A. Stevens, J. E. Fischer et al., "Molecular characterization of novel and selective peroxisome proliferatoractivated receptor alpha agonists with robust hypolipidemic activity in vivo," Molecular Pharmacology, vol. 75 , no. 2, pp. 296-306, 2009.

[13] S. M. H. Chan, X. Y. Zeng, R. Q. Sun et al., "Fenofibrate insulates diacylglycerol in lipid droplet/ER and preserves insulin signaling transduction in the liver of high fat fed mice," Biochimica et Biophysica Acta (BBA) - Molecular Basis of Disease, vol. 1852, no. 7, pp. 1511-1519, 2015.

[14] G. V. Ronnett, E. K. Kim, L. E. Landree, and Y. J. Tu, "Fatty acid metabolism as a target for obesity treatment," Physiology \& Behavior, vol. 85, no. 1, pp. 25-35, 2005.

[15] L. Napal, P. F. Marrero, and D. Haro, “An intronic peroxisome proliferator-activated receptor-binding sequence mediates fatty acid induction of the human carnitine palmitoyltransferase 1A," Journal of Molecular Biology, vol. 354, no. 4, pp. 751759, 2005.

[16] N. Price, F. van der Leij, V. Jackson et al., "A novel brainexpressed protein related to carnitine palmitoyltransferase I," Genomics, vol. 80, no. 4, pp. 433-442, 2002.

[17] J. D. McGarry and N. F. Brown, "The mitochondrial carnitine palmitoyltransferase system. From concept to molecular analysis," European Journal of Biochemistry, vol. 244, no. 1, pp. 1$14,1997$.
[18] I. R. Schlaepfer and M. Joshi, "CPT1A-mediated fat oxidation, mechanisms, and therapeutic potential," Endocrinology, vol. 161, no. 2, 2020.

[19] J. M. Orellana-Gavalda, L. Herrero, M. I. Malandrino et al., "Molecular therapy for obesity and diabetes based on a longterm increase in hepatic fatty-acid oxidation," Hepatology, vol. 53, no. 3, pp. 821-832, 2011.

[20] S. F. Han, J. Jiao, W. Zhang et al., "Lipolysis and thermogenesis in adipose tissues as new potential mechanisms for metabolic benefits of dietary fiber," Nutrition, vol. 33, pp. 118-124, 2017.

[21] M. Schweiger, R. Schreiber, G. Haemmerle et al., "Adipose triglyceride lipase and hormone-sensitive lipase are the major enzymes in adipose tissue triacylglycerol catabolism," The Journal of Biological Chemistry, vol. 281, no. 52, pp. 4023640241, 2006.

[22] M. J. Watt, A. G. Holmes, S. K. Pinnamaneni et al., "Regulation of HSL serine phosphorylation in skeletal muscle and adipose tissue," American Journal of Physiology-Endocrinology and Metabolism, vol. 290, no. 3, pp. E500-E508, 2006.

[23] W. D. van Marken Lichtenbelt, J. W. Vanhommerig, N. M. Smulders et al., "Cold-activated brown adipose tissue in healthy men," The New England Journal of Medicine, vol. 360, no. 15, pp. 1500-1508, 2009.

[24] M. S. Hedrington and S. N. Davis, "Peroxisome proliferatoractivated receptor alpha-mediated drug toxicity in the liver," Expert Opinion on Drug Metabolism \& Toxicology, vol. 14, no. 7, pp. 671-677, 2018.

[25] A. Liu, K. W. Krausz, Z. Z. Fang, C. Brocker, A. Qu, and F. J. Gonzalez, "Gemfibrozil disrupts lysophosphatidylcholine and bile acid homeostasis via PPAR $\alpha$ and its relevance to hepatotoxicity," Archives of Toxicology, vol. 88, no. 4, article 1188, pp. 983-996, 2014. 